\title{
SNAPPE-II and risk of neonatal morbidities in very low birth weight preterm infants
}

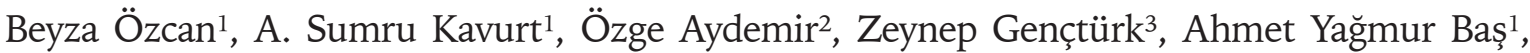 \\ Nihal Demirel ${ }^{1}$ \\ ${ }^{1}$ Department of Neonatology, Etlik Zübeyde Hanım Women's Health Training and Research Hospital, Ankara; ${ }^{2}$ Department \\ of Neonatology, Osmangazi University Faculty of Medicine, Eskişehir; ${ }^{3}$ Department of Statistics, Ankara University Faculty \\ of Medicine, Ankara, Turkey. E-mail: drbeyzaozcan@gmail.com \\ Received: 6th December 2016, Revised: 2nd January 2017, Accepted: 4th February 2017
}

SUMMARY: Özcan B, Kavurt AS, Aydemir Ö, Gençtürk Z, Baş AY, Demirel N. SNAPPE-II and risk of neonatal morbidities in very low birth weight preterm infants. Turk J Pediatr 2017; 59: 105-112.

Illness severity scores were described to estimate the mortality and morbidity risks based on data obtained shortly after admission to an intensive care unit. The aim of this study is to evaluate Scores for Neonatal Acute PhysiologyPerinatal Extension-II (SNAPPE-II) as a predictors of neonatal morbidities such as bronchopulmonary dysplasia (BPD) and retinopathy of prematurity (ROP). A retrospective cohort study was conducted between October 20112012. A total of 278 preterm infants born with gestational age (GA) $<32$ weeks and birthweight (BW) $<1,500 \mathrm{~g}$ were given SNAPPE-II scores based on data collected within the first $12 \mathrm{~h}$ of admission to ICU. The main outcomes were the development of BPD and ROP. The main variable was the SNAPPE-II obtained at admission. Logistic regression and receiver-operating characteristics (ROC) curve were calculated for SNAPPE-II. The mean GA and mean BW of the whole cohort were 29.2 weeks $( \pm 2.15)$ and $1,323 \mathrm{~g}$ $( \pm 331,4)$, respectively. The median SNAPPE-II value was significantly higher among patients with BPD and ROP. After logistic regression the SNAPPE-II was independent risk factor for BPD and ROP. The best discriminative cutoff value for BPD was 14.5 (sensitivity 92\%) and for ROP was 23.5 (sensitivity $80 \%$ ). The present study reviewed an association between SNAPPE-II and neonatal morbidities including ROP and BPD.

Key words: bronchopulmonary dysplasia, neonatal morbidities, prematurity, retinopathy of prematurity, SNAPPE-II.

The increased survival of very low birth weight (VLBW) infants due to recent advances in neonatal care has produced a new population of infants at very high risk of developing neonatal morbidities. The severity of the disease at hospitalization contributes significantly to the outcome of the patients. Illness severity scores are designed to estimate the clinical status of the patients ${ }^{1,2}$. Moreover, measurement of illness severity is central to make fair comparisons of outcomes of different neonatal intensive care units (NICUs) ${ }^{3}$. The Score for Neonatal Acute Physiology-Perinatal ExtensionII (SNAPPE-II) is a scoring system developed and validated by Richardson et $\mathrm{al}^{4}$. in 2001 for illness severity and mortality risk scores for
NICU. SNAPPE-II is intended for use at all gestational ages ${ }^{5}$. Severity of illness among very preterm newborns is not only associated with mortality, but also with neonatal morbidities, such as intraventricular hemorrhage, chronic lung disease and retinopathy of prematurity. This should not come as a surprise since some early neonatal characteristics of scores such as SNAPPE-II overlap with characteristics/ correlates of neonatal morbidity. The risk prediction at admission is also important while informing the parents about future outcomes. Studies were designed to assess the contribution of the SNAPPE-II as predictor of mortality and morbidity in newborns ${ }^{6,7}$.

The SNAPPE -II has been adopted as standard 
Table I. Postnatal Variables Included in the SNAPPE-II Illness Severity Score.

\begin{tabular}{|c|c|c|}
\hline Variables & Category & Scores \\
\hline \multirow[t]{3}{*}{ Mean blood pressure } & $>29 \mathrm{~mm} \mathrm{Hg}$ & 0 \\
\hline & $20-29 \mathrm{~mm} \mathrm{Hg}$ & 9 \\
\hline & $<20 \mathrm{~mm} \mathrm{Hg}$ & 19 \\
\hline \multirow[t]{3}{*}{ Corporal temperature } & $>35.6^{\circ} \mathrm{C}$ & 0 \\
\hline & $35-35.6^{\circ} \mathrm{C}$ & 8 \\
\hline & $<35^{\circ} \mathrm{C}$ & 15 \\
\hline \multirow[t]{3}{*}{ Birth weight } & $>999 \mathrm{~g}$ & 0 \\
\hline & $750-999 \mathrm{~g}$ & 10 \\
\hline & $<750 \mathrm{~g}$ & 17 \\
\hline \multirow[t]{2}{*}{ Small for gestational age } & No & 0 \\
\hline & Yes & 12 \\
\hline \multirow[t]{4}{*}{$\mathrm{PO}_{2} / \mathrm{FiO}_{2}$ ratio } & $>2.49$ & 0 \\
\hline & $1-2.49$ & 5 \\
\hline & $0.3-0.99$ & 16 \\
\hline & $<0.3$ & 28 \\
\hline \multirow[t]{3}{*}{ Lowest serum $\mathrm{pH}$} & $>7.19$ & 0 \\
\hline & $7.10-7.19$ & 7 \\
\hline & $<7.10$ & 16 \\
\hline \multirow[t]{3}{*}{ Urine output } & $>0.9 \mathrm{ml} / \mathrm{kg} / \mathrm{h}$ & 0 \\
\hline & $0.1-0.9 \mathrm{ml} / \mathrm{kg} / \mathrm{h}$ & 5 \\
\hline & $<0.1 \mathrm{ml} / \mathrm{kg} / \mathrm{h}$ & 18 \\
\hline \multirow{2}{*}{ Multiple seizures } & None & 0 \\
\hline & Yes & 19 \\
\hline \multirow{2}{*}{ Apgar score at $5 \mathrm{~min}$} & $>7$ & 0 \\
\hline & $<7$ & 18 \\
\hline
\end{tabular}

score in our NICU since September 2011. We have conducted a retrospective study to determine whether the SNAPPE-II parameters during the first 12 hours of life can predict neonatal morbidities such as bronchopulmonary displasia (BPD) and retinopathy of prematurity (ROP).

\section{Material and Methods}

\section{Study design and patients}

A retrospective cohort study was conducted in Etlik Zubeyde Hanim Women's Health Training and Research Hospital, Ankara, Turkey between October 2011 and October 2012. A total of 278 preterm infants born with gestational age (GA) $<32$ weeks and birthweight $(\mathrm{BW})<1,500$ $\mathrm{g}$ were enrolled. The patients with major congenital anomalies and neonates of GA < 24 weeks or BW $<400 \mathrm{~g}$ were not included in the study. SNAPPE-II were calculated based on data collected within the first $12 \mathrm{~h}$ of admission to NICU. We excluded 32 infants who died before the time to diagnose ROP and BPD. Complete clinical and eye examination data were available for 246 patients.

The main outcomes were the development of severe ROP and BPD. The main variable was the SNAPPE-II obtained at admission. SNAPPEII were calculated using 9 physiological and laboratory parameters (Table I). Clinical characteristics of the study population such as BW, GA, gender, mode of delivery, maternal chorioamnionitis, antenatal steroid, respiratory support (duration of mechanical ventilation, nasal continuous positive airway pressure and supplemental oxygen), respiratory distress syndrome (RDS), intraventricular hemorrhage (grade $>3$ ), sepsis, hemodynamically significant patent ductus arteriosus (PDA), BPD and severe ROP were recorded.

Gestational age was determined with prenatal ultrasound examination, or date of last menstrual period or The New Ballard. Small 
for gestational age (SGA) is defined as infants with a birth weight below the $10^{\text {th }}$ percentile for gestational age ${ }^{8}$. Chorioamnionitis was diagnosed by the presence of a fever of $38.3^{\circ} \mathrm{C}$ or higher, and the presence of at least two of the following: leukocytosis $\left(>18,000 / \mathrm{mm}^{3}\right)$, vaginal discharge with foul odor, uterine sensitivity, and tachycardia (maternal >100/ min, fetal $>180 / \mathrm{min})^{9}$. Sepsis was defined as proven when a blood culture or bacterial culture from a normally sterile site was positive for a likely pathogen and if bacterial cultures were negative, as highly probable in the presence of a clinical and biological systemic inflammatory response syndrome associated with infection. Hemodynamically significant PDA was defined according to echocardiography parameters. Left atrium-to-aortic root diameter ratio of $\geq 1.4$ in the parasternal long-axis view, ductal diameter of $\geq 1.4 \mathrm{~mm} / \mathrm{kg}$ body weight, left ventricular enlargement, and reversed diastolic flow in the descending aorta indicated a significant ductal shunt ${ }^{10}$. Antenatal steroid was considered to have been administered if betamethasone therapy was completed 24 hours before delivery. The first eye examination was performed at 4 weeks of chronologic age for infants born at

Table II. Demographic Features of the Patients.

\begin{tabular}{ll}
\hline Features & Results \\
\hline Birth weight* (g) & $1,323 \pm 331,4$ \\
Gestational age* (weeks) & $29.2 \pm 2.15$ \\
Gender, n (\%) & $121(49.2)$ \\
Female & $125(50.8)$ \\
Male & $52(21.1)$ \\
Delivery type, n (\%) & $194(78.9)$ \\
VD & $17(6.9)$ \\
C/S & $229(93.1)$ \\
Chorioamnionitis (\%) & $152(61.8)$ \\
Yes & $54(22.0)$ \\
No & $115(46.7)$ \\
Antenatal steroids, n (\%) & $131(53.3)$ \\
Yes & $5.5 \pm 1.35$ \\
No & $4.1 \pm 3.3$ \\
RDS, n (\%) & $18.3 \pm 1.6$ \\
Yes & \\
No & \\
Mespiratory support* (days) & $13(5.3)$ \\
Mechanical ventilation & $233(94.7)$ \\
OxAP & $27(11.0)$ \\
Oxygen therapy & $219(89.0)$ \\
IVH (\%) & $42(17.1)$ \\
Yes & $204(82.9)$ \\
No & $10(4.1)$ \\
Yes & $236(95.9)$ \\
No & $28(11.4)$ \\
PDA, n (\%) & $218(88.6)$ \\
Yes & No \\
Sovere ROP, n (\%) & \\
Yes & No \\
BPD, n (\%) & \\
Yes & No \\
SNAPPE-II & \\
\hline &
\end{tabular}

*: data is presented as mean \pm standard deviation). BPD: bronchopulmonary dysplasia; C/S: Caesarean section; IVH: intraventricular hemorrhage (grade $>3$ ), PDA: hemodynamically significant patent ductus arteriosus; RDS: respiratory distress syndrome; ROP: retinopathy of prematurity, severe ROP defined as ROP requiring treatment; VD: vaginal delivery. 
$\geq 27$ weeks or at 31 weeks of postmenstrual age for infants born at 24 to 26 weeks. All assessments for ROP were made by the same pediatric ophthalmologist throughout the study period. Staging of ROP was recorded according to the international classification of $\mathrm{ROP}^{11}$. The criteria for treatment were: zone I any stage of

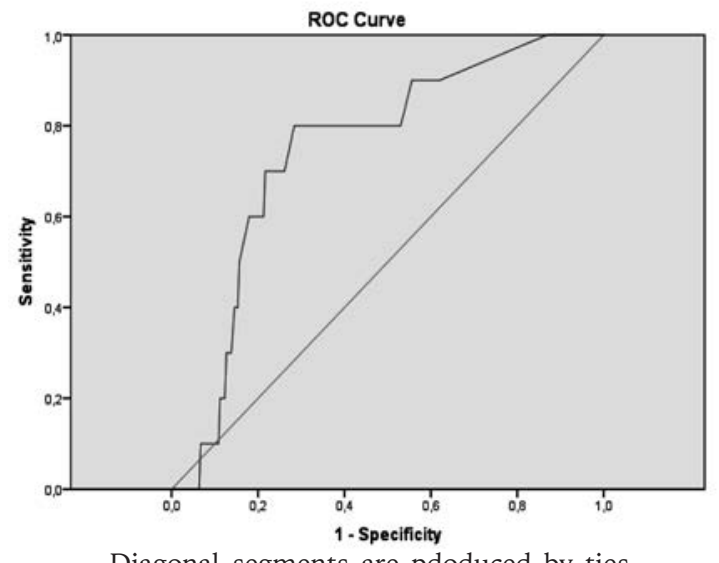

Diagonal segments are pdoduced by ties.
ROP with plus disease or zone I stage 3 without plus and zone 2 stage 2 or 3 with plus disease as defined by Early Treatment for Retinopathy of Prematurity Cooperative Group ${ }^{12}$; Severe ROP was defined as ROP requiring treatment. BPD was defined as oxygen requirement at 36-weeks postmenstrual age (PMA) for an infant who was born at $\leq 32$ weeks' gestation ${ }^{13}$. Intraventricular hemorrhage (IVH) was based

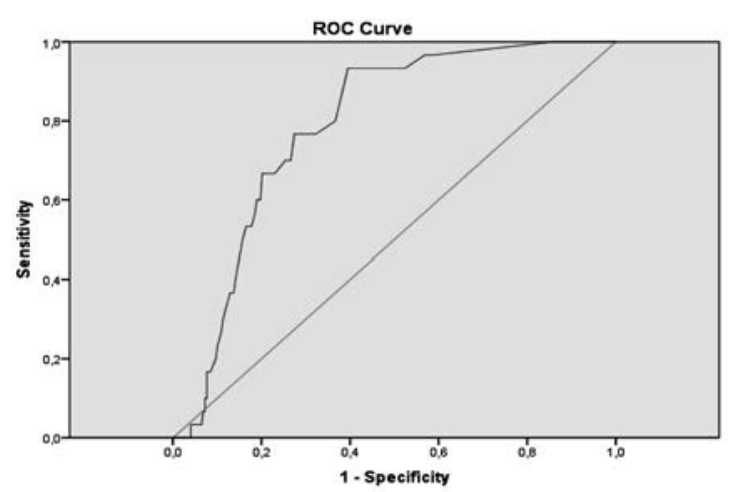

Diagonal segments are pdoduced by ties.

Fig. 1A and B. Receiver operating characteristic curves for the ability of the Score for Neonatal Acute Physiology Perinatal Extension-II (SNAPPE-II) to predict the development of ROP and BPD. The area under the ROC curve for SNAPPE-II for ROP (A) and BPD (B) were 0.81(CI 95\% 0.67-0.95) and 0.85 (CI 95\% 0.78-0.91), respectively.

Table III. Risk Factors for Retinopathy of Prematurity in the Whole Cohort (Univariate Analysis).

\begin{tabular}{llll}
\hline Risk Factors & No/mild ROP (n:236) & Severe ROP (n:10) & P \\
\hline $\begin{array}{l}\text { Birth weight* (g) } \\
\text { Gestational age (weeks) }\end{array}$ & $1410 \pm 306.3$ & $969 \pm 284$ & $<0.001$ \\
$\begin{array}{l}\text { Gender, n (\%) } \\
\text { Female }\end{array}$ & $117(23-35)$ & $27(24-30)$ & $<0.001$ \\
Male & $129(52.4)$ & $4(40)$ & 0.413 \\
Delivery type, n (\%) & $36(21.0)$ & $6(60)$ & \\
VD & $200(79.0)$ & $3(30.0)$ & 0.742 \\
C/S & $13(7.0)$ & $7(70.0)$ & 0.907 \\
Chorioamnionitis, n (\%) & $156(57.8)$ & $1(10.0)$ & 0.038 \\
Antenatal steroids, n (\%) & $8(80.0)$ & $<0.001$ \\
Respiratory support* (days) & $1.1 \pm 2.96$ & & $<0.001$ \\
Mechanical ventilation & $2.53 \pm 3.82$ & $8.13 \pm 12.69$ & $<0.001$ \\
CPAP & $10.63 \pm 15.82$ & $9.55 \pm 7.52$ & 0.002 \\
Oxygen therapy & $77(41.2)$ & $9(90.0)$ & 0.045 \\
RDS, n (\%) & $180(96.3)$ & $10(100.0)$ & $<0.001$ \\
IVH, n (\%) & $32(11.9)$ & $5(50.0)$ & $<0.001$ \\
Sepsis, n (\%) & $23(8.3)$ & $7(70.0)$ & $<0.001$ \\
BPD, n (\%) & $61(22.8)$ & $6(60.0)$ & $<0.001$ \\
PDA, n (\%) & $9(0-71)$ & $34.5(5-63)$ & . \\
SNAPPE-II &
\end{tabular}

*: data is presented as mean \pm standard deviation). BPD: bronchopulmonary dysplasia; C/S: Caesarean section; IVH: intraventricular hemorrhage (grade>3), PDA: patent ductus arteriosus; RDS: respiratory distress syndrome; VD: vaginal delivery. 
Table IV. Adjusted Odds Ratio for Retinopathy of Prematurity Development as a Function of the SNAPPEII at Admission (Logistic regression adjusted for gestational age, birth weight, SNAPPE-II, occurrence of sepsis, BPD, PDA, respiratory support, RDS, IVH, use of antenatal steroids).

\begin{tabular}{llll}
\hline Risk Factors & OR & $95 \%$ CI & P \\
\hline BPD & 15.274 & $2.954-78.982$ & 0.001 \\
SNAPPE-II & 1.050 & $1.004-1.099$ & 0.001 \\
\hline
\end{tabular}

BPD: bronchopulmonary dysplasia; IVH: intraventricular hemorrhage; PDA: patent ductus arteriosus; RDS: respiratory distress syndrome;

on cranial US evaluation in the first week of life according to Volpe criteria ${ }^{14}$.

\section{Statistical analysis}

Statistical analysis was conducted using the SPSS version 17.0 (SPSS Inc., Chicago, IL). The results are presented as numbers (n), frequencies (\%), means with respective standard deviation (SD) and medians. Non parametric tests were used to analysis continuous variables. Chi-square test was used to compare categorical variables. Logistic regression analysis was done to determine independent risk factors for BPD and ROP. Statistical significance was accepted $\mathrm{p}<0.05$.

The study was approved by Institutional
Ethics Committee (Report number:13/2014) and informed consent was obtained from the parents of the infants.

\section{Results}

There were 246 patients in this study. The mean GA and mean BW of the whole cohort were 29.2 weeks $( \pm 2.15)$ and $1,323 \mathrm{~g}$ $( \pm 331,4)$, respectively. Table II shows the demographic and clinical characteristics of the study population.

Infants with ROP had significantly lower BW, GA but higher incidence of sepsis, PDA, BPD, surfactant requiring treatment considered as RDS, respiratory support (duration of mechanical ventilation, nasal continuous

Table V. Risk Factors for Bronchopulmonary Dysplasia in the Whole Cohort (Univariate Analysis).

\begin{tabular}{|c|c|c|c|}
\hline Risk Factors & $\begin{array}{l}\text { BPD } \\
(\mathrm{N}: 28)\end{array}$ & $\begin{array}{l}\text { Without BPD } \\
(\mathrm{N}: 218)\end{array}$ & $\mathrm{P}$ \\
\hline Birth weight (g) & $945.7 \pm 271.2$ & $1371.5 \pm 306$ & $<0.001$ \\
\hline Gestational age (weeks) & $26(24-31)$ & $30(23-35)$ & $<0.001$ \\
\hline $\begin{array}{l}\text { Gender, n (\%) } \\
\text { Female } \\
\text { Male }\end{array}$ & $\begin{array}{l}16(57.1) \\
12(42.9)\end{array}$ & $\begin{array}{l}105(48.2) \\
113(51.8)\end{array}$ & 0.371 \\
\hline $\begin{array}{l}\text { Delivery type, n (\%) } \\
\text { VD } \\
\text { C/S }\end{array}$ & $\begin{array}{l}8(28.6) \\
20(71.4)\end{array}$ & $\begin{array}{l}44(20.2) \\
174(79.8)\end{array}$ & 0.306 \\
\hline Chorioamnionitis, n (\%) & $3(10.7)$ & $14(6.4)$ & 0.421 \\
\hline Antenatal steroids, n (\%) & $22(78.6)$ & $130(59.6)$ & 0.142 \\
\hline $\begin{array}{l}\text { Respiratory support* (day } \\
\text { Mechanical ventilation } \\
\text { CPAP } \\
\text { Oxygen therapy }\end{array}$ & $\begin{array}{l}3.7 \pm 6.2 \\
7.5 \pm 7.82 \\
39.3 \pm 15.82\end{array}$ & $\begin{array}{l}2.1 \pm 5.0 \\
4.5 \pm 4.2 \\
9.8 \pm 3.82\end{array}$ & $\begin{array}{l}0.069 \\
0.067 \\
0.005\end{array}$ \\
\hline RDS, n (\%) & $25(89.3)$ & $90(41.3)$ & $<0.001$ \\
\hline $\mathrm{IVH}, \mathrm{n}(\%)$ & $5(17.9)$ & $8(3.7)$ & 0.009 \\
\hline Sepsis, n (\%) & $13(46.4)$ & $14(6.4)$ & $<0.001$ \\
\hline PDA, n (\%) & $22(78.6)$ & $36(16.5)$ & $<0.001$ \\
\hline SNAPPE-II & $32.96(5-70)$ & $9(0-71)$ & $<0.001$ \\
\hline
\end{tabular}

*: data is presented as mean \pm standard deviation). BPD: bronchopulmonary dysplasia; C/S: Caesarean section; IVH: intraventricular hemorrhage (grade>3), PDA: patent ductus arteriosus; RDS: respiratory distress syndrome; VD: vaginal delivery. 
Table VI. Adjusted for Bronchopulmonary Dysplasia Development as a Function of the SNAPPE-II at Admission (Logistic regression adjusted for gestational age, birth weight, SNAPPE-II, RDS, duration of supplemental oxygen requirement, occurrence of sepsis, PDA, IVH, and use of antenatal steroids).

\begin{tabular}{llll}
\hline Risk Factors & OR & $95 \%$ CI & P value \\
\hline Gestational age & 0.607 & $0.454-0.812$ & 0.001 \\
PDA & 3.597 & $1.116-11.593$ & 0.032 \\
SNAPPE-II & 1.044 & $1.013-1.076$ & 0.005
\end{tabular}

BPD: bronchopulmonary dysplasia; IVH: intraventricular hemorrhage; PDA: patent ductus arteriosus; RDS: respiratory distress syndrome.

positive airway pressure and supplemental oxygen) and higher SNAPPE-II (Table III). There was no significant difference observed in the antenatal steroid between the two groups (Table III). The variables with significance in our study were: BPD, and SNAPPE-II, after the logistic regression (Table IV). Duration of assisted ventilation and supplemental oxygen requirement were not independent risk factors for development of ROP.

Univariate analysis included 14 variables clinically involved in the development of BPD. The BPD group and non BPD group differed with respect to birth weight, gestational age, RDS, duration of supplemental oxygen requirement, sepsis, PDA and SNAPPE-II (Table $\mathrm{V})$. After logistic regression, the SNAPPE-II, GA and PDA were independent risk factors of BPD (Table VI). Duration of assisted ventilation and supplemental oxygen requirement were not independent risk factors for BPD.

The median SNAPPE-II value was significantly higher among patients with BPD, and with ROP; $p<0.001, p<0.001$, respectively. After logistic regression, the SNAPPE-II was an independent risk factor for BPD and ROP.

On the ROC curve analysis of gestational age < 32 weeks, the best discriminative cut-off value of SNAPPE-II was 14.5 for BPD (sensitivity $92 \%$, specificity $68.3 \%$ ), and 23.5 for ROP (sensitivity $80 \%$, specificity $79 \%$; Fig. 1).

\section{Discussion}

Illness severity scores are important predictors of neonatal mortality and clinical outcomes. In most of the studies, various neonatal illness severity scores are compared with relationship to mortality among preterm babies. The increased survival of very low birth weight (VLBW) infants due to recent advances in neonatal care has produced a new population of infants at very high risk of developing comorbidity ${ }^{4}$. Previous articles have evaluated the usefulness of the neonatal illness severity scores like CRIB, SNAP, SNAPPE and cumulative SNAP, to predict mortality and morbidities including ROP and $\mathrm{BPD}^{15,16}$. In our study, high SNAPPE-II values showed better discrimination as predictors of neonatal morbidities and as a common independent risk factor for ROP and BPD.

Retinopathy of prematurity is frequently related to lower gestational age and birth weight, and the oxygen therapy. A previous study reported that none of the 4 tested systems (Neonatal Therapeutic Intervention System, CRIB, SNAP and SNAPPE) showed sufficient power to predict long-term outcomes, including visual impairment among ROP patients ${ }^{17}$. Fortes Filho et al. ${ }^{7}$ showed an association between higher SNAPPE-II values at admission and development of ROP among VLBW preterm babies by univariate analysis, but after adjusted logistic regression, as well as after the ROC curve results, the SNAPPE-II at admission did not enhance the assessment of risk for ROP. In another study, cumulative neonatal illness severity was shown as an independent risk factor for progression from moderate to severe ROP, but after adjustment for different risk factors, the cumulative SNAP system values did not enhance the assessment of risk for ROP ${ }^{18}$. Our results suggested an association between higher SNAPPE-II values at admission and the development of severe ROP after univariate analysis and logistic regression.

The study by Asker et al. ${ }^{19}$ which was conducted in 5 different centers in Turkey, has showed that the usage of antenatal steroid increases the predictive value of the SNAPPE-II score. However, we did not find any correlation in our study. 
Bronchopulmonary dysplasia is the most common serious pulmonary morbidity in premature infants. The increasing rates of survival among the smallest of premature infants due to changes in antenatal and postnatal respiratory management, such as antenatal steroids and surfactant replacement, contribute to an overall increase in the incidence of BPD despite advances in the care of premature infants that reduce the risk or severity of BPD. Yanhong et al. ${ }^{20}$ has investigated the association between SNAP and the development of BPD. In that study, high SNAP scores were significantly associated with increased risk for BPD or death in critically ill preterm infants with less than 33 gestational weeks. SNAP was predictive of BPD or death, with additional prognostic value when used in conjunction with other perinatal risk factors. Another study evaluated the usefulness of SNAP version II (SNAP-II) to predict BPD, suggesting SNAP-II predicts chronic lung disease in the NICU. They have suggested that SNAP- II should be used in combination with other risk variables for quantifying risk in outcome comparison ${ }^{21}$. To the best of our knowledge, the present study is first report for the relationship between SNAPPE-II and risk of BPD. The predictive value of SNAPPE-II in critically ill preterm infants in relationship to BPD is currently unknown. In our study, the SNAPPE-II was an independent risk factor for BPD and every one point each increase in the SNAPPE-II value increased to risk of BPD 1.04 times (sensitivity 92\%, specificity 68.3\%).

The present study had several limitations. The results might be biased due to the small sample size and should be confirmed in a multicenter study. Also, SNAPPE- II may measure not only admission illness severity but also interventions during the first 12 hours of NICU admission. The time interval for data collection may influence the prediction of scores. This limitation is intrinsic to all physiologic scores such as SNAP and CRIB.-

Higher SNAPPE-II values indicate patients with higher morbidities by NICU admission, but sometimes some of these sicker babies can reach a stable clinical course during their hospital stay period. On the other hand, less sick babies with lower SNAPPE-II values at admission can develop a worse clinical course during the same period. In these babies, severe neonatal morbidities can occur. Future studies are needed to show that SNAPPE-II should be combined with clinical course to reach a better predictive model.

In conclusion; we have shown an association between higher SNAPPE-II and neonatal morbidities among the immature newborns. Neonatal severity of illness scores should be further evaluated in cohorts of preterm infants and larger studies are needed to further explore the role of these scores for prediction of morbidities in preterm infants.

\section{REFERENCES}

1. The CRIB (Clinical Risk Index for Babies) score: a tool for assessing initial neonatal risk and comparing performance of neonatal intensive care units. The International Neonatal Network. Lancet 1993; 342: 193-198.

2. Fleisher BE, Murthy L, Lee $S$, et al. Neonatal severity of illness scoring systems: A comparison. Clin Pediatr (Phila) 1997; 36: 223-227.

3. Pollack MM, Koch MA, Bartel DA, et al. A comparison of neonatal mortality risk prediction models in very low birth weight infants. Pediatrics 2000; 105: 10511057.

4. Richardson DK, Corcoran JD, Escobar GJ, et al. SNAPII and SNAPPE-II: Simplified newborn illness severity and mortality risk scores. J Pediatr 2001; 138: 92-100.

5. Zupancic JA, Richardson DK, Horbar JD, et al. Revalidation of the Score for Neonatal Acute Physiology in the Vermont Oxford Network. Pediatrics 2007;119: e156-e163.

6. Dammann O, Shah B, Naples $\mathrm{M}$, et al. SNAP-II and SNAPPE-II as predictors of death among infants born before the 28th week of gestation. Inter-institutional variations. Pediatrics 2009; 124: e1001-e1006.

7. Fortes Filho JB, Dill AJC, Ishizaki A, et al. Score for Neonatal Acute Physiology and Perinatal Extension II as a predictor of retinopathy of prematurity: Study in 304 very-low-birth-weight preterm infants. Ophthalmologica 2009; 223: 177-182.

8. Battaglia FC, Lubchenco LO. A practical classification of newborn infants by weight and gestational age. J Pediatr 1967; 71: 159.

9. Kim YM, Romero R, Chaiworapongsa T, et al. Toll-like receptor- 2 and -4 in the chorioamniotic membranes in spontaneous labor at term and in preterm parturition that are associated with chorioamnionitis. Am J Obstet Gynecol 2004; 191: 1346.

10. Hamrick SE, Hansmann G. Patent ductus arteriosus of the preterm infant. Pediatrics 2010; 125: 1020.

11. The International Committee for the Classification of Retinopathy of Prematurity. The International Classification of Retinopathy of Prematurity Revisited. Arch Ophthalmol 2005; 123: 991-999. 
12. Early Treatment for Retinopathy of Prematurity Cooperative Group. Revised indications for the treatment of retinopathy of prematurity: Results of the early treatment for retinopathy of prematurity randomized trial. Arch Ophthalmol 2003; 121: 16841694.

13. Trembath A, Laughon MM. Predictors of bronchopulmonary dysplasia. Clin Perinatol 2012; 39: $585-601$

14. Volpe JJ. Intracranial hemorrhage: Germinal matrix intraventricular hemorrhage of premature infant. In: Volpe JJ (ed). Neurology of Newborn (4th ed). Philadelphia, PA: Saunders, 2001: 428-493.

15. Zardo MS, Procianoy RS. Comparison between different mortality risk scores in a neonatal intensive care unit. Rev Saude Publica 2003; 37: 591-596.

16. Silveira RC, Schlabendorff M, Procianoy RS. Predictive value of SNAP and SNAPPE for neonatal mortality. J Pediatr (Rio J) 2001; 77: 455-460.
17. Eriksson M, Bodin L, Finnstrom O, Schollin J. Can severity-of-illness indices for neonatal intensive care predict outcome at 4 years of age? Acta Paediatr 2002; 91: 1093-1100.

18. Hagadorn JL, Richardson DK, Schmid $\mathrm{CH}$, Cole $\mathrm{CH}$ Cumulative illness severity and progression from moderate to severe retinopathy of prematurity. J Perinatol 2007; 27: 502-509.

19. Asker HS, Satar M, Yıldızdas H, et al. Evaluation of Score for Neonatal Acute Physiology and Perinatal Extension II and Clinical Risk Index for Babies with additional parameters. Pediatr Int 2016; 58: 984-987.

20. Yanhong Li, Jie Yan, Mengxia Li, et al. Addition of SNAP to perinatal risk factors improves the prediction of bronchopulmonary dysplasia or death in critically ill preterm infants. BMC Pediatr 2013; 13: 138.

21. Chien LY, Whyte R, Thiessen P, et al. SNAP-II predicts severe intraventricular hemorrhage and chronic lung disease in the neonatal intensive care unit. J Perinatol 2002; 22: 26-30. 